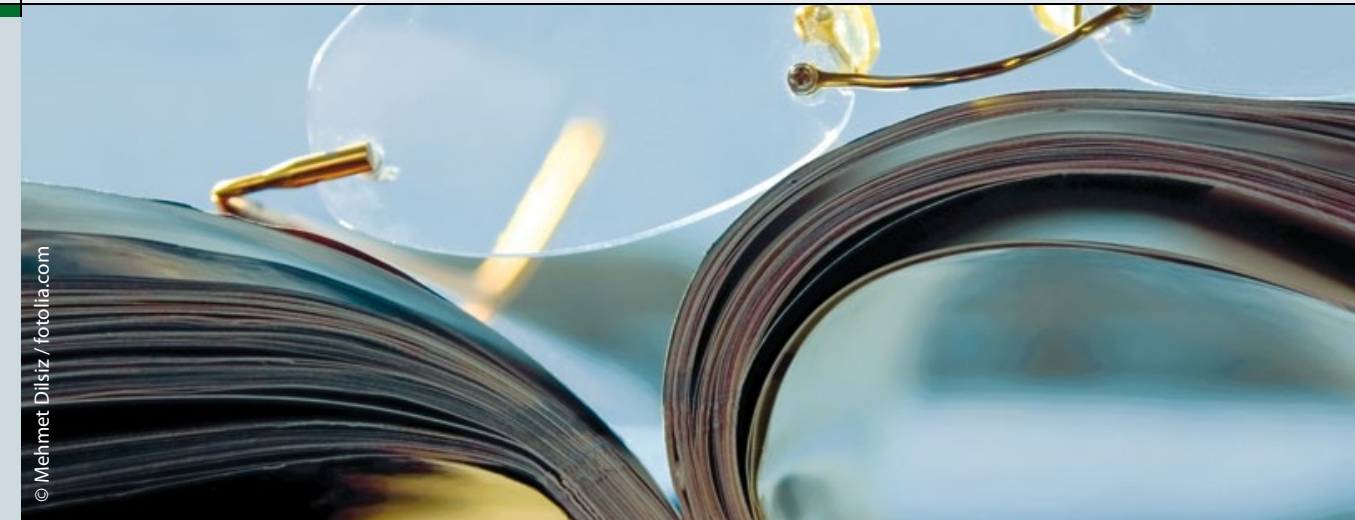

\title{
Die häufigsten Komplikationen bei perkutaner Nephrolithotomie
}

Eine positive Urinkultur vor einer perkutanen Nephrolithotomie verdoppelt das Risiko für postoperatives Fieber. Ob der Patient fiebert oder nicht, hängt zudem von der Art der Keime ab, die präoperativ nachgewiesen worden sind.

$\mathrm{D}$ ie perkuante Nephrolithotomie (PCNL) ist eine beliebte Methode, um Steine aus der Niere zu entfernen, vor allem wenn diese größer oder zahlreich sind. Das Verfahren gilt als schnell und risikoarm. Dennoch tritt postoperativ immer wieder Fieber bei den Patienten auf. Um die Gründe hierfür näher zu untersuchen, wurden Daten von 5.354 Patienten mit PCNL aus 96 Zentren ausgewertet. Von allen Patienten lag eine präoperative Urinkultur vor und sie hatten eine Antibiotikaprophylaxe zur Vermeidung einer Urosepsis erhalten.

Bei 16,2\% der untersuchten Patienten ergab sich eine positive Urinkultur. Unter den Keimen führte international Escherichia coli mit 40,5\% die Reihe an, gefolgt von Proteus (11\%), Enterococcus (8,2\%), Pseudomonas (8,0\%), Klebsiella (7,5\%), Staphylococcus $(7,2 \%)$ und anderen. Bei $10 \%$ der Proben ergaben sich Mischkulturen. Betrachtete man Europa gesondert, blieben die ersten drei Plätze gleich, Kleb-

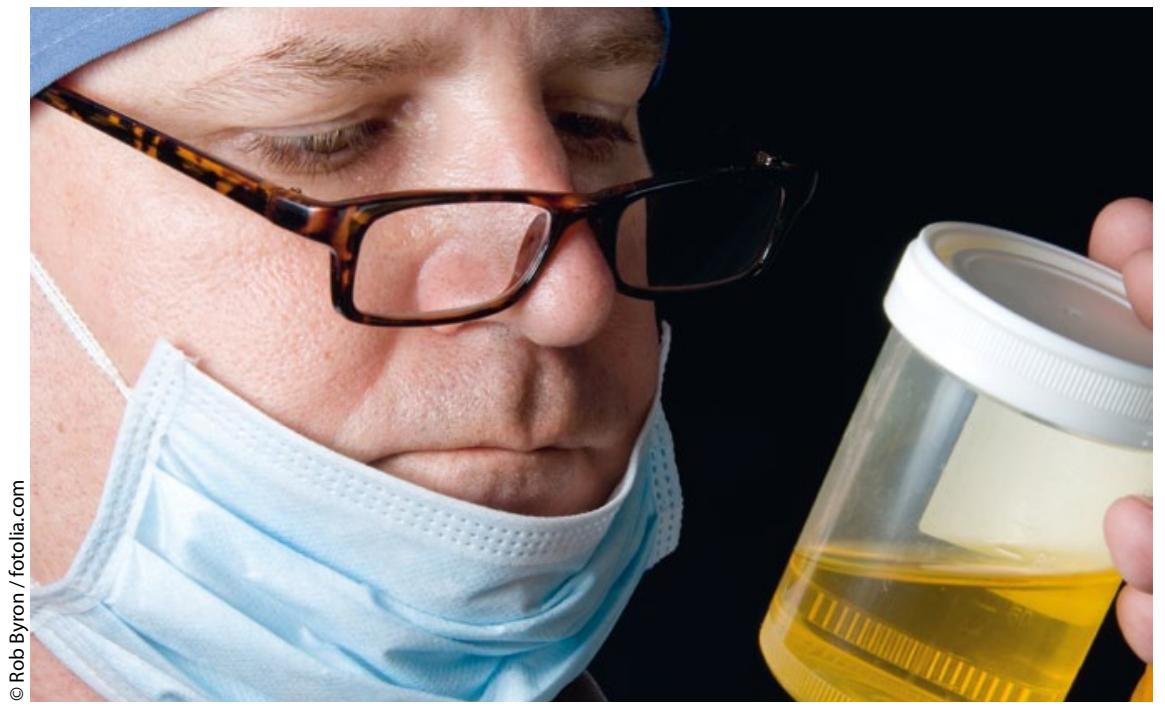

Eine positive Urinkultur ist nur einer der Risikofaktoren für postoperatives Fieber nach Nierenstein-Operation. siella trat etwas häufiger $(8,3 \%)$ und Pseudomonas etwas seltener (6,2\%) auf. 10,4\% der operierten Patienten entwickelten nach dem Eingriff Fieber $\left(>38,5^{\circ} \mathrm{C}\right)$. Die Temperatur stieg vor allem bei Patienten mit postoperativ positiver Urinkultur (18,2\% vs. 8,8\% mit negativer Urinkultur vor der PCNL). Dabei fiel auf, dass die Patienten, bei denen in der Urinkultur gramnegative Keime nachgewiesen worden waren, deutlich häufiger fieberten als Patienten mit grampositiven (z.B. 23,8\% bei Enterobacter vs. 9,7 \% bei Staphylococcus).

Insgesamt ergab sich in der Multivarianzanalyse im Zusammenhang mit folgenden Faktoren ein erhöhtes Riskio für postoperatives Fieber: positive Urinkultur, Nierenkelchsteine, präoperative Nephrostomie, jüngeres Alter und Diabetes.

Fazit: Fieber ist bei einem Harnwegsinfekt eine übliche Begleiterscheinung (21$39,8 \%$ ). So ist es erstaunlich, dass in der aktuellen Studie nur 10,4\% der Patienten fieberten. Dies kann, so die Autoren, an verschiedenen Messzeiten oder auch an der unterschiedlichen Definition von Fieber liegen. Denn in manchen Studien wird bereits eine Temperatur von $38^{\circ} \mathrm{C}$ als Fieber gewertet. Dass trotz einer Antibiotikaprophylaxe Harnwegsinfektionen aufgetreten sind, zeigt, dass diese Maßnahme das Infektionsrisiko im Zusammenhang mit einer PCNL nicht vollständig ausschalten kann, sei es aufgrund von Resistenzen oder auch wegen eines falschen Antibiotikums.

Dr. Christine Starostzik

Gutierrez J et al. Urinary tract infections and post-operative fever in percutaneous nephrolithotomy. World J Urol 2012; doi: 10.1007/s00345-012-0836-y 